\title{
Dominância de membros tomadores ou poupadores de recursos nas cooperativas de crédito e 0 desempenho: análise sob a ótica da teoria de agência
}

\author{
Mário Nazzari Westrup Mestre em Desenvolvimento Socioeconômico. Universidade do Extremo Sul Catarinense (Unesc) - Brasil. \\ mario.westrup@w3ge.com \\ Sílvio Parodi Oliveira Camilo Pós-Doutor em Ciências Contábeis e Doutor em Administração. Universidade do Extremo Sul Catarinense \\ (Unesc) - Brasil. parodi@unesc.net \\ Dimas de Oliveira Estevam Doutor em Sociologia Política. Universidade do Extremo Sul Catarinense (Unesc) - Brasil. doe@unesc.net
}

\section{RESUMO}

Possíveis conflitos de interesses em cooperativas de crédito têm instigado pesquisas, dada a proliferação desse modelo de agremiação no Brasil e no resto do mundo, procurando compreender características dos grupos influentes e os impactos no desempenho. 0 objetivo geral desta pesquisa foi analisar em que medida a dominância - entendida como o poder de decisão seja aos membros tomadores ou poupadores de recursos -, afeta o desempenho das cooperativas de crédito. Este estudo integra problemas de agência entre principalprincipal às concepções do cooperativismo. A centralidade das discussões está no potencial conflito entre tomadores e poupadores de crédito e o seu efeito no desempenho. Da revisão da literatura foram propostas duas hipóteses no sentido de avaliar o relacionamento entre dominação e desempenho. Como estratégia de pesquisa foi realizado levantamento de dados de cooperativas filiadas ao Sistema de Crédito Cooperativo SICREDI. Para atingir o objetivo da pesquisa adotou-se como técnica a análise multivariada de dados, com o uso de dados em painel de 39 cooperativas filiadas ao SICREDI, no período de 2008 a 2014. Os dados apontaram que o índice de dominância é fator influente e estatisticamente significativo sobre o Retorno sobre os Ativos (ROA). Outra contribuição relevante foi a identificação da dominação por tomadores e a ausência de cooperativas neutras, 0 que pode violar a real função das cooperativas de crédito, sua sustentabilidade e o seu comprometimento com os princípios norteadores do cooperativismo, afastando-se assim de seus pressupostos, culminando em práticas contraditórias ao seu discurso.

Palavras-chave: Principal-principal. Desempenho nas Cooperativas. Conflito de interesses. Análise de dominação. Índice de dominância. 


\title{
Dominance of members takers or resource savers in credit cooperatives and performance: analysis under the perspective of agency theory
}

\begin{abstract}
Possible conflicts of interest in credit cooperatives have instigated research, given the proliferation of this model of association in Brazil and the rest of the world, seeking to understand characteristics of influential groups and the impacts on performance. The overall objective of this research was to analyze the extent to which dominance - understood as the decision-making power of borrowers or resource savers - affects the performance of credit cooperatives. This study integrates problems of agency between principal-principal and conceptions of cooperativism. The centrality of the discussions lies in the potential conflict between borrowers and credit savers and their effect on performance. From the literature review, two hypotheses have been proposed in order to evaluate the relationship between domination and performance. As research strategy was carried out data collection of cooperatives affiliated to the Cooperative Credit System SICREDI. In order to reach the objective of the research, multivariate data analysis was used as a technique with the use of panel data from 39 cooperatives affiliated to SICREDI, from 2008 to 2014. The data indicated that the dominance index is an influential factor and statistically significant return on assets (ROA). Another relevant contribution was the identification of domination by borrowers and the absence of neutral cooperatives, which may violate the real function of credit cooperatives, their sustainability and their commitment to the guiding principles of cooperativism, thus moving away from their assumptions, culminating in practices contradictory to his discourse.
\end{abstract}

Keywords: Principal-principal. Cooperative Performance. Conflict of interests. Analysis of do mination. Dominance index. $^{1}$ 


\section{INTRODUÇÃO}

Diferentemente de organizações empresariais, nas coo perativas de crédito os pontos de conflitos estão centrados na relação propriedade e controle (BLACK; DUGGER, 1981), ou seja, principal-principal (JENSEN; MECKLING, 1976), pois uma característica marcante das cooperativas de crédito é a relação um membro um voto.

As cooperativas também são tradicionalmente financiadas pela poupança dos cooperados. No entanto essas mesmas forças podem facilmente se tornar impedimentos para uma governança eficaz que as cooperativas de crédito precisam para expandir e competir no mercado financeiro.

No cooperativismo, que tem por princípio o mutualismo, especificamente no cooperativismo de crédito, que se caracteriza por promover acesso a serviços financeiros e intermediação de recursos privados assumindo riscos correspondentes e gerando benefícios para a comunidade, também têm questões específicas associadas à sua governança, que devem ser adequadamente tratadas (CRÚZIO, 2009). As cooperativas de crédito são diferentes em duas frentes: os conselheiros são eleitos pelo voto democrático e os proprietários também são os clientes. Cada uma destas características distintivas das cooperativas de crédito possui seus próprios desafios (SOARES; MELO SOBRINHO, 2009).

Branch e Baker (1998); Zylbersztajn (1994; 2002); Bialoskorski Neto (2012); Bialoskorski Neto, Bassoso e Rezende (2012); e Trindade e Bialoskorski Neto (2012; 2014); enfatizam duas características limitadoras das cooperativas brasileiras no contexto da governança corporativa: a inexistência de separação da propriedade e controle; e a dupla natureza do cooperado, pois o cooperado é ao mesmo tempo proprietário e cliente.

Seguindo a mesma linha, Westley e Branch (2000); Cuevas e Fischer (2006); e Bressan, Braga e Bressan (2012) mencionam que o conflito entre tomadores (que pegam recursos emprestados) e poupadores (que emprestam recursos) nas cooperativas de crédito tem recebido relativamente pouca atenção por parte dos pesquisadores. Apesar disso, há evidências empíricas que este conflito pode ser significativo e ter contribuído para muitos dos fracassos encontrados nas cooperativas de crédito latino-americanas.

Patin e McNiel (1991) explicam que se uma maior proporção da renda líquida é distribuída como remuneração (dividendos) aos poupadores, indica dominância por esse grupo. Em contraponto, uma maior proporção da renda líquida distribuída como retorno de juros dos empréstimos, indica a dominação por tomadores. A diferenças dessas medidas, ajustada ao viés de tamanho da cooperativa, constitui o índice de dominância da cooperativa.

Neste âmbito, as cooperativas de crédito brasileiras, ainda, são pouco exploradas. A proposição se justifica por contribuir com evidências empíricas já existentes em outros países e por investigar cooperativas de crédito considerando o contexto Brasileiro.

Adquire importância também como contribuição adicional para academia e aos poucos estudos que integram o conflito de agência, o controle, a dominância e desempenho de cooperativas de crédito no Brasil, como os de Meurer (2006); Tosini e Bastos (2008); Lima, Araújo e Amaral (2008) e Bressan, Braga e Bressan (2012).

Dada a crescente participação na economia, e seu impacto no desenvolvimento local, identificar fatores que afetam o desempenho pode contribuir para compreender as heterogeneidades e homogeneidades dessas organizações coletivas. 0 presente estudo visa contribuir com avanço em suas relações e em novas direções de pesquisa. Como questão norteadora se deriva a seguinte indagação: Em que medida o tipo de dominância, seja por tomadores ou poupadores de recursos, influencia o desempenho das cooperativas de crédito?

\subsection{Objetivo Geral}

Considerando a importância deste objeto de estudo, o objetivo geral do trabalho é analisar como a dominância, seja por tomadores ou poupadores de recursos, influencia no desempenho das cooperativas de crédito.

\subsection{Objetivos Específicos}

Os objetivos específicos são:

- Identificar as características das cooperativas, se tomadoras ou poupadoras; determinar o 
índice de dominância entre os tomadores e poupadores de crédito;

- Analisar o relacionamento entre o índice de dominância e desempenho, mediante análise multivariada de dados.

Apósa seção introdutória, apresentam-se os antecedentes teóricos e empíricos sobre o tema, destacando breve histórico da teoria de agência, o problema do conflito entre principal-principal e a relação de dominância entre tomadores e poupadores nas cooperativas de crédito. Em seguida são apresentados os procedimentos metodológicos da pesquisa, a análise e discussão dos resultados empíricos, e por fim as conclusões do estudo.

\section{ANTECEDENTES DA LITERATURA E HIPÓTESES}

Nesta seção são discutidos os pressupostos da teoria de agência, a partir da segregação entre propriedade e controle, relacionando-os com o problema no cooperativismo entre tomadores e poupadores, gerando um conflito principal- principal. Finalmente, será a abordada a dominância de cooperados na condição de tomadores e/ou poupadores e o desempenho da cooperativa de crédito.

\subsection{Breve histórico dos pressupostos da teoria da agência}

O trabalho seminal de Adolf. A. Berle e Gardiner Means, intitulado The Modern Corporation and Private Property publicado em 1932, iniciou os debates modernos que resultaram na teoria da governança corporativa. A obra delineou a teoria da separação entre propriedade e controle na corporação moderna e os problemas causados por essa separação. Mais tarde, com a teoria de agência de Jensen e Meckling (1976) a propriedade ficou conhecida como "principal" e o controle como "agente", dando origem ao conflito principal-agente.

Em síntese, o proprietário entrega ao agente o poder de decisão da empresa. Porém, existe um potencial para conflitos de interesses entre os proprietários e os gestores quando a propriedade e o controle das corporações não se encontram, sendo que o agente, seja por buscar interesses próprios, incompetência, negligência ou má-fé, pode não agir em favor dos interesses dos proprietários.

Dessa forma, pode-se conjecturar que toda separação entre propriedade e controle gera desalinhamento de interesses. Em empresas de capital aberto, por exemplo, em que o controle é confiado a um corpo executivo, relata-se, na literatura, a ocorrência de um problema de agência em que interesses dos acionistas não refletem fielmente o comportamento dos dirigentes responsáveis pela gestão da empresa.

Em organizações empresariais, de uma maneira geral, potenciais conflitos de interesse entre os gestores (agente) responsáveis pela tomada de decisões e os proprietários (principal), que sofrem as consequências dessas decisões, são mitigados por meio de mecanismos internos e externos de governança. Já em cooperativas os pontos de conflitos podem ser encontrados em diversas formas, conforme a Figura 01.

Neste caso de uma cooperativa comercializadora de produtos o associado pode desempenhar dois papéis, o de investidor e o de produtor. Como Investidor, que forma o capital da cooperativa, ele se comporta como o principal em relação ao Conselho de Administração (Agente), pois o conselho trabalha para gerar e garantir resultados. 
Figura 01 - Relações de Agência nas Cooperativas

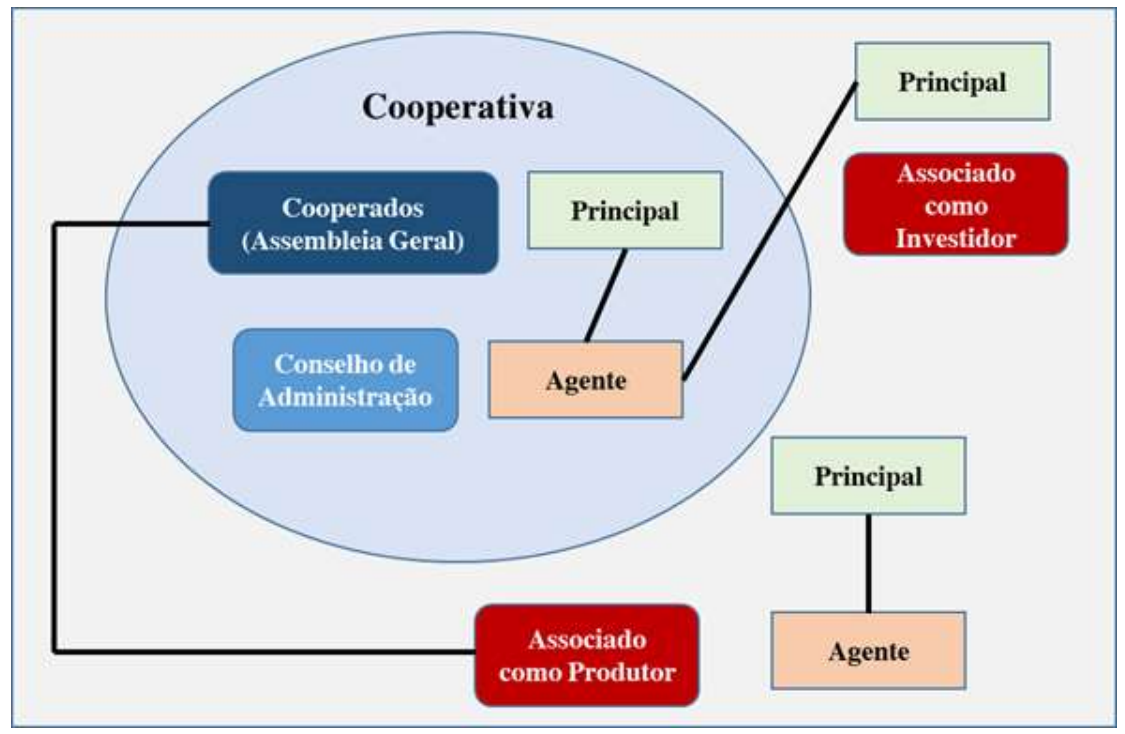

Fonte: Adaptado de Bialoskorski (2006).

Como produtor, o associado se torna Agente em relação a Cooperativa como um todo, dado que precisa produzir em quantidades e qualidade adequadas para atender os objetivos do negócio da cooperativa. Uma outra relação pode ser observada pela Assembleia de Cooperados como o Principal em face ao Conselho de administração como o agente, considerando que o conselho precisa ser incentivado e monitorado para cuidardos melhores interesses da cooperativa como um todo. Porém, nas cooperativas de crédito outro ponto de conflito pode estar centrado na relação entre membros tomadores e membros poupadores de crédito, onde todos os membros são proprietários (principal), delineando dessa forma um conflito principal-principal.

\subsection{Conflito principal-principal}

Os pesquisado res cada vez mais percebem que não há um único modelo de agência que adequadamente descreve os conflitos existentes em todos os contextos. 0 modelo predominante de governança para mitigar conflitos é um produto de economias desenvolvidas, principalmente Estados Unidos e Reino Unido. Nas economias desenvolvidas, como a propriedade e o controle são muitas vezes separados e os mecanismos legais protegem os interesses dos proprietários, os conflitos de governança que recebe maior parte das atenções são entre o principal e o agente (MORCK; SHLEIFER; VISHNY, 1987).

Os conflitos principal-principal são caracterizados pela propriedade concentrada e controle, fraca proteção institucional dos acionistas minoritários e poucos indicadores de governança nas empresas do país, tais como menos empresas de capital aberto; poucas informações contidas nos preços das ações; pouco investimento em inovação; e, em muitos casos, a expropriação dos acionistas minoritários (LA PORTA; LOPEZ-DE-SILANES; SHLEIFER, 1999). Na última década, pesquisadores em finançase economia têm cada vez afirmado que a conceituação tradicional do conflito principal- agente de Jensen e Meckling (1976) não representa as realidades dos conflitos principal-principal, que dominam as economias emergentes (YOUNG, 2008).

0 conflito principal-principal também pode ocorrer em economias desenvolvidas. Porém, a literatura indica grande parte de estudos subsequentes ao de Young (2008) em diversas economias emergentes onde as instituições são consideradas fracas, como em Singapura (WOAN, 2007), na China (SU; XU; PHAN, 2008; LI; QIAN, 2012), na Índia (PALEPU, 2009), no Leste Europeu (RENDERS; GAEREMYNCK, 2012), no Brasil e inclusive economias asiáticasem crise (WARD; FILATOTCHEV, 2010; J IANG; PENG, 2010; PENG; SAUERWALD, 2012).

Com base em Lima, Araújo, Amaral (2008), a partir do conflito de agência, se compara como esse problema é caracterizado nas empresas tradicionais e nas cooperativas de crédito. Por exemplo, vê-se que na relação entre tomador de empréstimo e poupador na cooperativa de crédito, há interesses difusos. Em razão disso, o gestor sofre pressões para empreender decisões favoráveis aos interesses do tomador e do poupador.

Como apontado por Westley e Branch (2000), Cuevas e Fischer (2006) e Bressan, Braga e Bressan (2012), há um campo extenso a ser explorado investigando a natureza do conflito entre tomadores e poupadores. Pois, 
estudos preliminares sugerem efeitos negativos no desempenho das cooperativas de crédito devido as relações conflituosas entre principal-principal.

As cooperativas de crédito também possuem grupos de principais. Porém, diferentemente das empresas tradicionais, não existe dominância de grupos controladores contra grupos minoritários, já que ela se baseia pelo princípio de um membro, um voto. Contudo, esse conflito pode se dar pelo diferencial no capital investido, e o interesse de determinados grupos em tomar ou poupar crédito. Políticas de distribuição de recursos a tomadores e remuneração a poupadores pode gerar conflito entre grupos (RUBIN et al, 2013).

0 grupo poupador se beneficia pelo fato de que os excedentes são devolvidos aos membros sob a forma de maiores retornos de poupança, enquanto o grupo tomador espera menores taxas de empréstimos. Dessa maneira, os excedentes são utilizados para o desenvolvimento da cooperativa e benefício dos membros (PARKER et al., 2014). Entretanto, o equilíbrio de interesses nem sempre é mantido afetando o princípio cooperativo da participação econômica dos sócios,

Destaca-se que o gestor da cooperativa (tomador de decisões) tanto pode estar na condição de tomador quanto de poupador. 0 tomador de recursos a fim de realizarseus projetos tem a pretensão de pagar o menor custo possível. Enquanto que o poupador ao alocar recursos excedentes pretende que os mesmos sejam remunerados por taxas dentro de sua expectativa. Assim, evidencia-se que a relação pode não ser parcimoniosa e equitativa, sujeita a contradições e, por sua vez, sensível a produção de conflitos com implicações na forma de dominância de grupos de interesses.

\subsection{Dominância de membros tomadores ou poupadores e desempenho da cooperativa}

A motivação fundamental de uma cooperativa de crédito é oferecer serviços financeiros a seus membros, em particular, um depósito de poupança e um acesso a crédito ao consumidor. No entanto existem uma série de razões pelas quais as cooperativas de crédito podem não chegar a um perfeito equilíbrio no tratamento dos tomadores e dos poupadores. Manter as taxas de empréstimo baixas podem reduzir a capacidade da cooperativa para oferecer taxas de dividendos elevadas, enquanto a manutenção de taxas de dividendos altas pode exigir taxas de empréstimo mais elevadas (LIMA; ARAÚj O; AMARAL, 2008).

Consequentemente, a competição desses dois objetivos estratégicos pode resultar no surgimento de conflitos entre os membros de cooperativas de crédito, que de um lado são poupadores e do outro são tomadores. Sendo os membros das cooperativas de crédito por sua vez os proprietários, o conflito poderá potencialmente resultar em um tratamento preferencial para um determinado grupo, orientando a cooperativa para o grupo de tomadores ou poupadores, em vez de um tratamento equilibrado ou neutro (BRESSAN; BRAGA; BRESAN, 2012).

$O$ conflito de agência que existe entre os membros tomadores e poupadores está relacionado ao fato de que ambos os tipos de membros podem ser representados no conselho de administração de uma cooperativa de crédito comum e, portanto, são capazes de influenciar o processo de decisão em seu benefício.

Com os trabalhos de Taylor (1971, 1977 e 1979) se observou teoricamente o possível conflito entre tomadores e poupadores dentro das organizações cooperativas crédito, sob o prisma da teoria econômica da cooperação aplicada a essa tipologia de organização. Os trabalhos de Walker e Chandler (1977), Smith (1986), Kohers e Mullis (1990), Patin e McNiel (1991), Legget e Stewart (1999) e Goddard e Wilson (2005), abordam a temática de avaliação de cooperativas de crédito, levando em consideração o aspecto de dominância, e elencam fatores que poderiam afetar o desempenho da cooperativa.

O frame disposto na Figura 02 demonstra a participação dos cooperados no Conselho de Administração, como mecanismo interno de governança, com atividades de monitoramento e controle sobre ações do gestor, por meio da avaliação de desempenho da cooperativa. 
Figura 02 - Design teórico

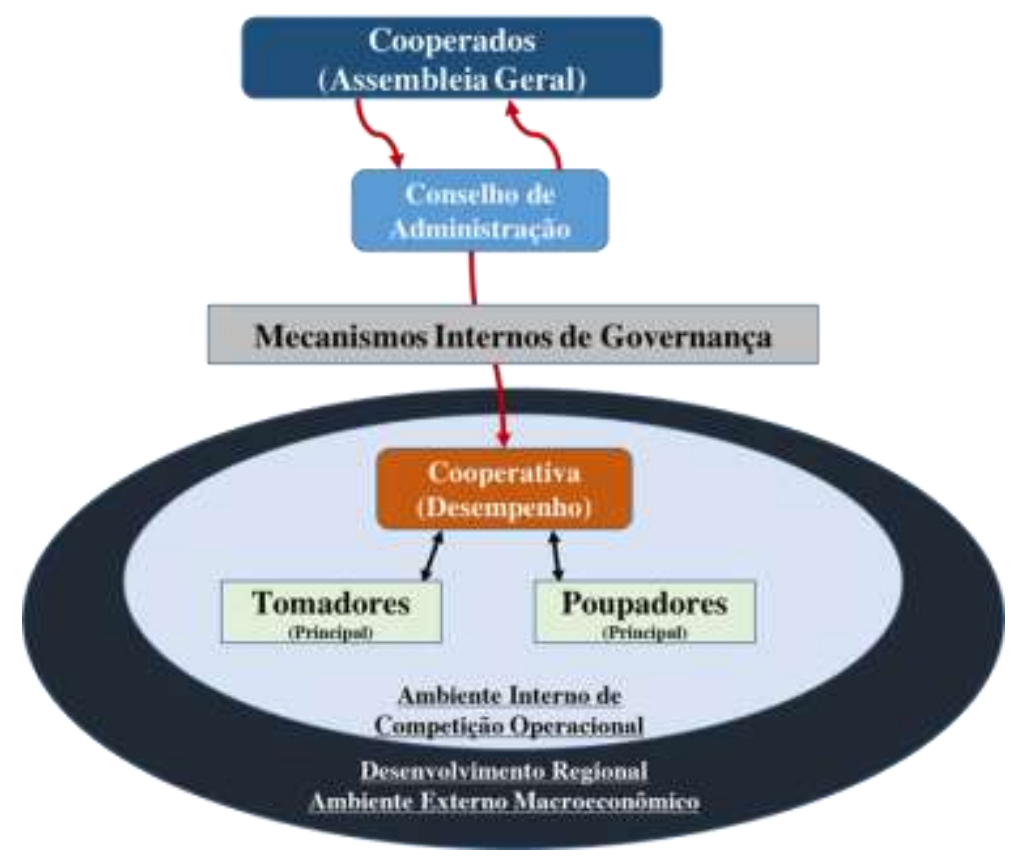

Fonte: Elaborado pelos autores.

Black e Dugger (1981) afirmam que o conflito entre esses dois interesses pode afetar o desempenho da cooperativa, e observam que as cooperativas de crédito podem ser classificadas por dominação de grupos tomadores ou poupadores, sendo que estas características podem impactar os meios de gestão e os seus resultados. Muitas cooperativas de crédito na América Latina lutam com problemas de governança que podem minar a saúde financeira e eficiência operacional, e até mesmo levar à falência. Esses problemas ocorrem quando os interesses dos tomadores superam os interesses dos poupadores (BRANCH; BAKER, 1998).

A extensa literatura suporta a noção de que o tipo de dominação da cooperativa de crédito afeta 0 desempenho. Por isso a importância de avaliar os conflitos e do minância de membros nas cooperativas de crédito, visando compreender fatores que podem afetar o desempenho dessas instituições que interferem no ambiente de forma ampla. A partir da literatura e antecedentes teóricos, se permitem delinear as seguintes hipóteses (H1 e H2) a serem testadas:

H1: Existe um relacionamento significativo entre 0 índice de dominação e 0 desempenho das cooperativas de crédito.

H2: Existe um relacionamento positivo entre o índice de dominação e o desempenho das cooperativas de crédito.

\section{PROCEDIMENTOS METODOLÓGICOS}

Esta seção apresenta primeiramente o enquadramento metodológico da pesquisa, na sequência é apresentado o objeto de estudo da pesquisa, e discorrido sobre o modelo de regressão com dados em painel, e sua variáveis.

\subsection{Enquadramento metodológico}

Pesquisas teóricas e empíricas podem ter o propósito de desenvolverem estruturas, sejam formulando hipóteses ou proposições, a serem testadas (WHETTEN, 1989). Este estudo se caracteriza como hipotético- 
dedutivo (FERNANDEZ; BÊRNI, 2012), justamente por que o objetivo principal será o de formular e testar hipóteses a partir de estudos teóricos e empíricos.

Sparrowe e Mayer (2011) sugerem que toda hipótese deve emergir de concepções teóricas e empíricas e formulada de maneira clara e lógica, relacionando variáveis possíveis de testabilidade. Nesse sentido, o presente trabalho apresentou um resgate de estudos teóricos e empíricos, donde a partir dessa base se desenvolveram duas hipóteses a serem testadas no campo da governança das cooperativas de crédito.

Como estratégia se caracteriza como uma pesquisa de levantamento de dados secundários. Isso porque resgata um conjunto de dados quantitativos, e os utiliza como base, e focaliza em acontecimentos contemporâneos (SAMPIERI; COLLADO; LUCIO, 2013).

Em se tratando dos fins, classifica-se a tal pesquisa como descritiva, pois de acordo com o que confirma Diehl e Tatim (2004, p. 53) "um projeto têm esta característica quando utilizado para conhecer melhor um determinado problema ou desafio, proporcionando melhor familiaridade com o tema".

\subsection{Objeto de estudo e modelo de regressão com dados em painel}

Para atingir os objetivos da pesquisa se obteve acesso às demonstrações financeiras de 39 cooperativas singulares, representando 33,0\% do sistema SICREDI- Brasil, e 84,7\% das atuantes nos estados do Rio Grande do Sul e Santa Catarina, que totalizavam 46 em fevereiro de 2017. Delas foram extraídas 7.371 informações anualizadas, referentes ao período de janeiro de 2008 a dezembro de 2014 (07 anos) e tabuladas em painel.

Para a avaliação da existência de dominação nas cooperativas de crédito é preciso encontrar os valores para os benefícios monetários líquidos distribuídos aos membros poupadores (BMLP) e aos membros tomadores (BMLT). Para isso será ajustado o modelo proposto por Patin e McNiel (1991) a realidade da economia e cooperativas brasileiras, conforme segue:

BMLP $=($ MTPA-MTMPA) $*$ TA

Onde':

$B M L P=$ benefícios monetários líquidos recebidos pelos poupadores.

MTPA = média das taxas pagas pela cooperativa de crédito sobre todos os instrumentos de aplicação oferecidos. Diante da impossibilidade de coletar diretamente nas cooperativas os valores referentes às taxas pagas pelas cooperativas sobre o montante de recursos, utilizou-se como proxy, a razão entre as despesas pagas pela cooperativa com as aplicações dos coo perados em reais e o total aplicado pelos cooperados em reais.

MTMPA = média das melhores alternativas de taxas pagas pelo mercado sobre os tipos similares de instrumentos de aplicação. Nesta variável, utilizou-se a seguinte proxy: SELIC - rendimento nominal - (\% a.a.). Essa escolha se justifica pelo motivo de este representar o custo de oportunidade.

$\mathrm{TA}=$ Volume total das aplicações (depósitos) realizadas pelos membros na cooperativa, em reais.

O BMLT pode ser medido pela diferença entre a taxa de empréstimo das cooperativas de crédito e a melhor alternativa de taxa de empréstimo no mercado, multiplicado pelo nível de empréstimos concedidos. A taxa sobre empréstimo da cooperativa deve também ser ajustada de acordo com a restituição de juros para os tomadores, que também tem direito dos resultados gerados das operações de concessão de crédito, conforme segue:

\footnotetext{
2 O manual de Oslo, desenvolvido em parceria pelo Eurostat e a OCDE, é um documento que tem como objetivo oferecer diretrizes para a coleta e interpretação de dados sobre inovação. A primeira edição do Manual de Oslo ocorreu em 1992 e foi referência para a pesquisa Community Innovation Survey (CIS) conduzida pela União Europeia. Os resultados desta culminaram na segunda edição do Manual, em 1997, na qual foi atualizada a estrutura de conceitos, definições e metodologia, expandindo o tratamento ao setor de serviços. Em razão do conceito de inovação tecnológica de produtos e de processo (TPP) não ser adequado ao setor de serviços, houve outra revisão no manual, dando origem a terceira edição, de 2005, que introduz dois novos tipos de inovação, a organizacional e de marketing (OCDE, 2005).
} 
BMLT $=\left(\right.$ MTEMC-MTEC $\left.\left(1-T X \_R E S T\right)\right) * T E$

Onde:

BMLT = benefícios monetários líquidos recebidos pelos tomadores.

MTEMC = média das taxas de empréstimos de mercado cobrada por outras instituições sobre instrumentos de dívida similares àqueles oferecidos pelas cooperativas. Nessa variável, utilizou-se a seguinte proxy: Taxa média anual das operações de crédito com recursos livres referenciais para taxa de juros - pessoas físicas/crédito pessoal - \% a.a., que são informações disponíveis à população.

MTEC = média das taxas de empréstimos cobrada pela cooperativa para todos os tipos de empréstimos aos seus membros. Novamente, como no caso de MTEC, utilizou-se a seguinte proxy: [rendas obtidas pela cooperativa (ou taxa cobrada pela cooperativa) sobre os empréstimos em reais / total de empréstimos concedidos em reais].

TX_REST = proporção da renda de juros sobre empréstimos que é restituída aos tomadores da cooperativa. Nesta variável utilizou-se a seguinte proxy: [renda restituída em reais / rendas obtidas pela cooperativa (ou taxa cobrada pela cooperativa) sobre os empréstimos].

$\mathrm{TE}=$ média entre anos do volume total de empréstimos concedidos aos membros da cooperativa, em reais.

Para comparar os benefícios alocados para cada grupo de membros é preciso calcular, para cada cooperativa de crédito, a diferença (DIF) entre os benefícios BMLP e BMLT:

$$
\mathrm{DIF}=\mathrm{BMLP}-\mathrm{BMLT}
$$

Seguindo uma lógica matemática básica, a cooperativa de crédito aloca mais benefícios para seus membros poupadores se o "DIF" da cooperativa de crédito for positivo. 0 valor de DIF $=0$ indica que a cooperativa de crédito aloca igualitariamente os benefícios entre ambos os grupos de membros. Se o "DIF" da cooperativa por negativo, a cooperativa aloca mais benefícios para seus membros tomadores.

Calculando o "DIF " para todas as cooperativas de crédito e comando $\Sigma$ DIF, é possível determinar se 0 sistema de cooperativas analisado aloca o maior nível de benefícios para os membros poupadores $(\Sigma D I F>0)$ ou para membros tomado res $(\Sigma \mathrm{DIF}<0)$, porém se o somatório indicar neutralidade $(\Sigma \mathrm{DIF}=0)$ não se pode dizer que 0 sistema é neutro, sendo este um conjunto de cooperativas tomadores, poupadoras e neutras (BRESSAN; BRAGA; BRESSAN, 2012).

Para ajustar o viés de tamanho da cooperativa, os autores Patin e McNiel (1991) sugerem um cálculo do nível em que cada cooperativa de aloca benefícios entre os membros no período $t$ analisado, por meio do que denominaram de índice de dominação (IDit), demonstrado na equação 4:

$$
\begin{aligned}
& \text { IDit }=\frac{\text { DSit }}{\underline{\underline{\text { STDt }}}}= \\
& \text { DSit }=\frac{\text { BMLPit }}{\underline{\text { TAit }}-\frac{\text { BMLTit }}{\text { TEit }}}
\end{aligned}
$$

$$
\text { STDt }=\text { Desvio padrão de DSit em torno de } 0
$$

Bressan, Braga e Bressan (2012 p. 11) interpretaram o modelo de Patin e McNiel (1991) onde afirma que "para a i-ésima cooperativa de crédito, DSit representa a diferença entre o BMLPit por real poupado" (TA = Total de Aplicações) e "BMLTit por real emprestado (TE = Total de Empréstimos), e STDt é o desvio padrão de DSit em torno de zero no tempo $t^{\prime \prime}$. Dessa forma, o valor absoluto de IDit fornece a o desvio para a i-ésima cooperativa de crédito - em relação ao comportamento neutro (IDit $=0$ ) no período t. 0 seu sinal indica se a cooperativa é dominada por poupadores (IDit $>0$ ) ou tomadores de crédito (IDit <0).

Com a obtenção do ROA está dada a variável dependente no modelo de regressão com dados em painel balanceado. Na sequência se utilizada o Índice de dominação (ID) recomendado por Patin e McNiel (1991). A partir dessas informações, pode-se classificar as variáveis como independentes e de controle, conforme o Quadro 01. 
Quadro 01 - Classificação das variáveis

\begin{tabular}{|c|c|c|}
\hline Variável & Classificação & Autores \\
\hline ROA (Retorno sobre o Ativo) & Dependente & Evans e Branch (2002) \\
\hline ID (Índice de Dominância) & Independente & Patin e McNiel (1991) \\
\hline DESPAT (Despesa operacional / Ativo) & Independente & Leggett e Stewart (1999) \\
\hline INAD (Taxa de inadimplência) & Independente & Leggett e Stewart (1999) \\
\hline LN_IDADE (Tempo de existência) & $\begin{array}{c}\text { Independente e de } \\
\text { Controle }\end{array}$ & $\begin{array}{c}\text { Smith (1986); Kohers e Mullis } \\
(1990) \text { e Goddard e Wilson (2005) }\end{array}$ \\
\hline LN_RESCAP (Reserva de Capital / Ativo) & $\begin{array}{c}\text { Independente e de } \\
\text { Controle }\end{array}$ & $\begin{array}{c}\text { Walker e Chandler (1977); Smith } \\
(1986) .\end{array}$ \\
\hline
\end{tabular}

Fonte: Elaborado pelos autores (2017).

Com a formação da variável dependente e a classificação das variáveis independentes e de controle, é realizada a tabulação dos dados em painel para a aplicação do modelo de regressão. Uma razão para usar agrupamentos de cortes transversais é aumentar o tamanho da amostra. Ao agrupar amostras aleatórias extraídas do mesmo objeto, mas em períodos de tempos diferentes, pode-se obter estimadores mais precisos e estatísticas mais poderosas (WOOLDRIDGE, 2011).

Dentre as principais vantagens de usar painéis de dados, Baltagi (1995) elenca que os dados em painel podem detectar e medir efeitos melhor do que quando a observação é feita por meio de corte transversal puro ou série temporal pura.

Os dad os em painel, também chamados de dados longitudinais, possuem duas dimensões: espacial (corte transversal) e temporal. Quando cada objeto dispõe do mesmo número de dados temporais, o painel caracterizase como balanceado. No presente estudo, utilizam-se demonstrações financeiras das cooperativas de crédito filiadas SICREDI-Brasil, e fornecem informações temporais para todo o período do estudo.

Para capturar os efeitos das variáveis independentes no indicador de desempenho (ROAit) foram testados, por análise de dados em painel, o método dos efeitos fixos (fixed effects - FE) e de efeitos aleatórios (random effects - RE). 0 método EF considera efeitos não observáveis que podem estar associados a variável dependente, mensurando-a em uma escala de tempo, sendo ela linearmente dependente de um conjunto de variável preditora (ALISSON, 2009).

A estratégia geral utilizada para selecionar a melhor especificação do modelo de dados em painel a ser utilizado, com intuito de avaliar os determinantes do índice de dominação nas cooperativas, consistiu em estimar primeiro os efeitos fixos. No segundo passo, apesar de não estar associado a natureza do problema, também se estima o modelo com efeitos aleatórios.

0 terceiro passo consiste em testar, conforme recomendado por Wooldridge (2011), se o modelo com efeitos aleatórios é preferível ao modelo com efeitos fixos, utilizando-se o teste de Hausman. 0 teste de especificação de Hausman é um teste estatístico que avalia a consistência de um estimador comparado a um outro estimador alternativo. Indicando $p$-valor significativo $(<0,05)$ deve-se considerar os efeitosfixos (GUJ ARATI; PORTER,2011). A estrutura básica do modelo de regressão em painel utilizada nesta pesquisa é dada por:

$$
\begin{aligned}
& \mathrm{ROA}_{i t}=\alpha+\beta I D I D_{i t}+\beta D_{\text {ESPATDESPAT }}+\beta I N A D I N A D_{i t}+\beta \text { LN_RESCAPLN_RESCAP }_{\text {it }}+ \\
& \beta L{ }_{-} \text {IDADELN_IDADEit }+v i+\varepsilon i \mathrm{t}
\end{aligned}
$$

Em que: $i=1, \ldots, 39$ cooperativas filiadas ao SICREDI-BRASIL; $t=1, \ldots, 8$ anos, referentes ao período de janeiro de 2008 a dezembro de 2014 das cooperativas filiadas ao SICREDI-BRASIL; ROA = retorno sobre 0 ativo da cooperativa, e constitui a variável dependente do modelo; $\alpha=$ é o intercepto do modelo; $\beta=$ representa os coeficientes angulares estimados para cada variável independente, que são: ID = Índice de dominação; DESPAT = 
Despesas operacionais sobre 0 ativo total médio; INAD = Taxa de inadimplência; LN_RESCAP = Reserva de capital; LN_IDADE = Tempo de existência da cooperativa, em anos; e $v i+\varepsilon i t=$ são os resíduos do modelo. 0 software utilizado para o processamento do modelo de dados foi o Stata ${ }^{\circledR}$ V. 12, ferramenta da StataCorp @ Texas, Estados Unidos.

As amostras de dados LN_IDADE (idade) e LN_RESCAP (Reserva de Capital) mostram significante diferença. Portanto foram utilizados os dados logaritmizados, a fim de eliminar a anormalidade na distribuição de dados (HAIR et at. 2009).

\section{ANÁLISE E DISCUSSÃO DOS RESULTADOS EMPíRICOS}

Considerando o exame da variável ROA, em razão do pressuposto da pesquisa, o método dos efeitosfixos (FE) foi o mais adequado. A Tabela 01 apresenta os influenciadores do desempenho (ROA).

De acordo com a Tabela 01, as variáveis ID, INAD e LN_IDADE apresentaram

*significância a p-valor <0,05. As variáveis DESPAT e LN_RESCAP não apresentaram significância.

Tabela 01 - Influenciadores do Desempenho (ROA)

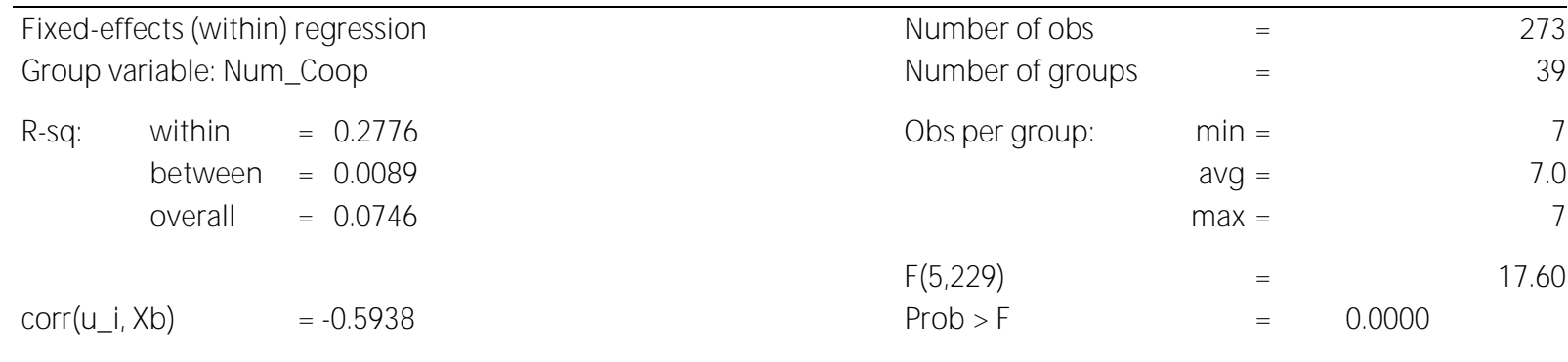

\begin{tabular}{|c|c|c|c|c|c|c|}
\hline ROA | & Coef. & Std. Err. & $\mathrm{t}$ & $P>|t|$ & [95\% Conf. Inter & \\
\hline ID | & .0048534 & .0011859 & 4.09 & $0.000 * * *$ & .0025168 & .00719 \\
\hline DESPAT| & .1523621 & .18099 & 0.84 & 0.401 & -.2042566 & .5089807 \\
\hline INAD | & -.3119815 & .0515302 & -6.05 & $0.000 * * *$ & -.4135155 & -2104476 \\
\hline Ln_Rescap | & .0021382 & .0022485 & 0.95 & 0.343 & -.0022922 & .0065686 \\
\hline Ln_Idade | & .0161393 & .0081404 & 1.98 & $0.049 *$ & .0000996 & .032179 \\
\hline cons & -.038039 & .0349112 & -1.09 & 0.277 & -.1068272 & .0307493 \\
\hline
\end{tabular}

\footnotetext{
sigma_u| $\quad .01656683$

sigma_el $\quad 01415004$

rho | $57819566 \quad$ (fraction of variance due to $u_{-} \mathrm{i}$ )

$\begin{array}{llll}\text { F test that all } \mathrm{u}_{-} \mathrm{i}=0: & \mathrm{F}(38,229)= & 3.71 & \text { Prob }>F=0.0000\end{array}$

Fonte: Resultados da pesquisa, 2017.

$$
\text { Nota: } * * *<.001,{ }^{* *} p<.01, * p<.05 \text { e } \quad+p<.10 \text {. }
$$

Com a sugestão de Patin e McNiel (1991) de criar um índice de dominação (ID), ajustado para o viés de tamanho e a medida do grau que a cooperativa aloca os benefícios para um grupo de membros específico, verificou-se no presente estudo que $98,9 \%$ das observações indicam uma diferença de benefícios entre tomadores e poupadores negativa, evidenciando que as cooperativas analisadas alocam mais benefícios para os membros tomadores de recursos.
} 
A partir disso, podemos afirmar que a variável ID sendo quase na tua totalidade negativa, temos uma amostra de coo perativas tomadoras de recursos, e ao avaliar a significância de impacto no ID no ROA, ela é positiva, e com alta significância. Ao resgatar as hipóteses $\mathbf{H 1}$ e $\mathbf{H 2}$ pode-se afirmar que há suporte para serem confirmadas. Portanto foram encontradas evidências no modelo do impacto da dominância no desempenho, o que gera contribuição aos estudos precedentes.

O resultado para a variável INAD, que representa a qualidade do ativo, ou seja, o montante de inadimplência em cima dos empréstimos concedidos, foi de influência negativa ao desempenho e com alta significância. Osautores LeggetteStewart(1999) quando levantaram essa medida não haviam obtido observações.

A variável LN_IDADE, afeta positivamente o ROA e se mostrou significante. Para Smith (1986), Kohers e Mullis (1990), Goddard e Wilson (2005) a idade representa uma maturidade da cooperativa, que caminha para maior estabilidade e para dominação de membros poupadores de crédito, conforme o seu envelhecimento.

Já a variável DESPAT, que também pode ser considerada como uma medida de eficiência, mostrando a relação entre as despesas operacionais da cooperativa e 0 ativo total, apresentou efeito positivo em relação ao ROA. Porém não apresentou significância, evidenciando que sua variação não é relevante para o indicador de desempenho do modelo.

A variável LN_RESCAP, formada pela relação de alavancagem da cooperativa, medindo o indicador de Reservas de Capital pelo Ativo Total, se mostrou positiva ao ROA, porém não significante. Os autores Walker e Chandler (1977) e Smith (1986) argumentaram que as cooperativas com este indicador positivo, representariam uma cooperativa com membros poupadores, e com indicador negativo, membros tomadores.

Analisando os dad os de origem (sem tratamento),98,9\% das cooperativas são tomadoras de crédito, e em nenhuma das 273 observações se apresentou uma relação negativa entre Reservas de Capital e o Ativo Total. Como implicações, a prevalência dominante de certo grupo pode gerar mais atratividade aos minoritários, importando em fortalecimento do mesmo (RUBIN et al., 2013). Isso pode revelar contradição em relação aos princípios do cooperativismo com segregação de interesses, por produzir excedentes a partir do grupo tomador em benefício do desenvolvimento da cooperativa, mas em benefício de membros poupadores (PARKER et al., 2014).

\section{CONCLUSÕES}

O objetivo geral desta pesquisa foi e é analisar como a dominância, seja por tomadores ou poupadores de recursos, influencia no desempenho das cooperativas de crédito. Detectou-se que o índice de dominação, a inadimplência e a idade da cooperativa são influentes e estatisticamente significativos, o que sugere implicações importantes sobre o ROA. De acordo com as evidências dessa amostra, podem-se confirmar as hipóteses H1 e H2.

Os resultados encontrados, como a dominância de membros tomadores em praticamente todas as cooperativas analisadas, e a ausência de cooperativas neutras, pode importar em indagação quanto a real função das cooperativas de crédito, sua sustentabilidade, e o seu comprometimento com os princípios norteadores do cooperativismo. Muitas cooperativas na tentativa de se manterem competitivas no mercado utilizam estratégias semelhantes as organizações empresariais, afastando- se assim de seus pressupostos, culminando em práticas contraditórias a seu discurso.

Um dos pressupostos da teoria econômica do cooperativismo afirma que os dirigentes atendem a seus próprios interesses na medida em quefomentam os interesses dos associados, o que gera a necessidade de se estabelecer o controle contra ações negativas dos dirigentes e também dos cooperados. Para mitigar os conflitos abordados pela teoria de agência, entre eles o conflito principal-principal, está a necessidade da adaptação de mecanismos de governança cooperativa para equilibrar interesses entre cooperados tomadores e poupadores.

0 modelo econométrico apresentado neste estudo pode revelar efeitos da dominação sobre a performance. Contribuir para compreender grupos homogêneos e heterogêneos de cooperativas de créditos e os impactos a partir de suas características de dominação. Se suas condições iniciais de cooperativismo se perpetuam e constituem condições de equilíbrio, mantendo valores para equidade coletiva.

Como limitações, o estudo não investiga benefícios não monetários e os mecanismos de governança cooperativa. 0 conselho de administração, como um desses mecanismos, é importante para administrar e mitigar as tensões entre gestores, tomadores e poupadores, de modo que as decisões sejam mais benéficas ao conjunto 
de cooperados e não imponham riscos a cooperativa de crédito. As deliberações e atas de conselho foram fatores inobserváveis nesta pesquisa, o que pode gerar interpretações incompletas.

Características das cooperativas apontam para o cooperado desempenhando papéis difusos, seja na qualidade de gestor, prop rietário ou cliente na condição de tomador e/ou poupador. A pesquisa quantitativa, com o modelo econométrico sendo uma simplificação da realidade, apesar de usar a fundamentação teórica para formular as variáveis, não captura todas essas caraterísticas e variáveis que interveem no objeto estudado. Produções brasileiras que abordem o tema ainda são escassas no Brasil, o que não permite maiores comparações, também limitando as análises e conclusões.

Considerando as limitações, ampliações e novas questões de pesquisa podem se delinear. Por exemplo: os resultados obtidos para este modelo devem ser confirmados por novos estudos considerando informações das cooperativas a partir de 2015. 0 Conselho Monetário Nacional (CMN), por intermédio da Resolução $n=4.434$, de 5 de agosto de 2015, estabeleceu novas regras sobre a constituição, as alterações estatutárias e o cancelamento de autorização para funcionamento das cooperativas de crédito, além de outras providências processuais e administrativas. Esta norma fixa um amplo conjunto de regras de governança e estruturais as cooperativas de crédito no Brasil.

A partir desta norma (Resolução no 4.434/2015), as cooperativas no país passaram a ser classificadas como: (i) plenas, que podem praticar todas as operações autorizadas ao setor; (ii) clássicas, que não operam com derivativos, variação cambial, securitização, empréstimo de ativos, operações compromissadas e cotas em fundos de investimento; e de (iii) capital e empréstimo, que operam sem captação, utilizando apenas os recursos aportados pelos associados. Testar o modelo avaliando o comportamento dos indicadores e influenciadores do índice de dominação, após esse marco regulatório, pode evidenciar novos resultados que indiquem caminhos ainda inexplorados, sugerindo pesquisas futuras.

Também se recomenda verificar se existem diferenças significativas entre os índices de dominação em outros sistemas de cooperação de crédito existentes no Brasil, como UNICRED, SICOOB, CECRED, entre outros. Neste sentido, pode-se analisar se o comportamento de dominância está comprometendo a sustentabilidade financeira destas cooperativas, que desempenham um papel importante no microcrédito e no desenvolvimento local.

Em relação ao desenvolvimento local, pode-se realizar pesquisas levando em conta a variável de localização geográfica, considerando o índice de dominância em municípios esvaentes (forte queda populacional) e atraentes (que tiveram crescimentos superiores aos seus estados). A distribuição de recursos pela cooperativa de crédito, pode acelerar e alavancar o crescimento de determinada região. Baseado nessa afirmação pode-se sugerir avaliar a distribuição de benefícios monetários e fatores de dominação nas cooperativas, ligados ao crescimento da localização geográfica.

Conflito de interesses dos participantes aliados a análise de um contexto ambiental, equilibrado ou turbulento, pode influenciar os movimentos, políticas ou arranjos de grupos. Investigações que abordem as decisões e relações de poder entre quotistas, seja na qualidade de membros do conselho ou até mesmo gestores, numa função dual ou trial se mostram importantes em futuros estudos.

A definição de boas práticas de governança em cooperativas de crédito envolve mecanismos que fortalecem suas estruturas e processos, de forma sistematicamente articulada, para ampliar as condições gerais de segurança, eficiência e a redução de riscos. Estudos empíricos analisando determinadas características das cooperativas e as relações políticas de grupo de interesses podem estar relacionados ao desempenho das cooperativas. 


\section{REFERÊNCIAS}

ALISSON, P. D. Fixed effects regression models. Los Angeles, California: Sage, 2009.

BALTAGI, B. H. Econometric Analysis of Panel Data. New Jersey: Wiley, 1995.

BERLE A.A.; MEANS G. C. The Modern Corporation and Private Property. New York: Macmillan, 1932.

BIALOSKORSKI NETO, S. Aspectos econômicos das cooperativas. Belo Horizonte: Mandamentos, 2006.

BIALOSKORSKI NETO, S. Economia e Gestão de Organizações Cooperativas. 2. ed. São Paulo: Atlas, 2012.

BIALOSKORSKI NETO, S.; BARROSO, M. F. G.; REZENDE, A. J. Governança cooperativa e sistemas de controle gerencial: uma abordagem teórica de custos da agência. Brazilian Business Review, Vitória, v. 9, n. 2, p. 72-92, jun. 2012.

BLACK, H.; DUGGER, R. H. Credit Union Structure, Growth and Regulatory Problems. The Journal of finance, Singapore, v. 36, n. 2, maio 1981.

BRANCH B.; BAKER C. Overcoming Credit Union Governance Problems: What Does It Take? Washington: InterAmerican Development Bank, 1998.

BRESSAN V. G. F.; BRAGA M. J.; BRESSAN A. A. Análise da dominação de membros tomadores ou poupadores de recursos nas cooperativas de crédito mineiras, Economia Aplicada, São Paulo, v. 16, n. 2, p. 339-359, jul. 2012.

CRÚZIO, H. de O. Governança Corporativa Financeira: nas cooperativas de crédito. São Paulo: Editora FGV, 2009.

CUEVAS, Carlos E., FISCHER, Klaus P. Cooperative financial institutions: issues in governance, regulation, and supervision. Washington: The World Bank, 2006.

DIEHL, A. A. TATIM, D. C. Pesquisa em ciências sociais aplicadas. São Paulo: Pearson Prentice Hall, 2004.

FERNANDEZ, B. P. M.; BÊRNI, D. A. Os Métodos da Ciência. In: BÊRNI, Duilio de Avila; FERNANDEZ, Brena Paula Magno. (Org.). Métodos e Técnicas de Pesquisa. São Paulo: Saraiva, 2012. p. 215-223.

GODDARD, J.; WILSON, J . O. S. US Credit Unions: An Empirical Investigation of Size, Age and Growth. Annals of Public and Cooperative Economics, United Kingdom, v. 76, n. 3, p. 375-406, 2005.

GUJ ARATI, D; PORTER, D.C. Econometria Básica. 5. ed. São Paulo: McGrawHill, 2011.

HAIR, J. F. et al. Análise multivariada de dados. 6. ed. Porto Alegre: Artmed, 2009.

JENSEN, M.; MECKLING, W. Theory of the firm: managerial behavior, agency costs and ownership structure. Journal of Financial Economics, Netherlands, v. 3, n. 4, p. 305-360, Oct. 1976.

JIANG, Y.; PENG, M. W. Principal-principal conflicts during crisis. Texas: Springer Science, 2010.

KOHERS, T.; MULLIS, D. The impact of a financial institution's age on its financial profile and operating characteristics: the evidence for the credit union industry. Review of Business and Economic Research, Australia, v. 26, p. 38-49, 1990.

LA PORTA, R.; LOPEZ-DE-SILANES, F.; SHLEIFER, A. Corporate Ownership around the World. The Journal of Finance, v. 54, n. 2, p. 472-479, Apr. 1999.

LEGGETT, K. J.; STEWART, Y. H. Multiple Common Bond Credit Unions and the Allocation of Benefits. Journal of Economics and Finance, United States, v. 23, n. 3, p. 235-245, 1999. 
LI, J.; QIAN, C. Principal-Principal Conflicts under Weak Institutions: A Studyof Corporate Takeovers in China. Strategic Management Journal, Singapore, v. 34, p. 498-508, jan. 2012.

LIMA, R. E.; ARAÚJ O M. B. V.; AMARAL H. F. Conflitos de agência: Um estudo comparativo dos aspectos inerentes a Empresas tradicionais e cooperativas de crédito. Belo Horizonte, MG:UFMG, 2008.

MEURER, Simplício. Desempenho da cooperativa versus expectativas e interesses dos associados do SICOOB/SC Creditapiranga. 2006. 104 f. Dissertação (Mestrado em Organizações e Sociedade) - Universidade do Vale do Itajaí, Biguaçu, 2006.

MORCK, R.; SHLEIFER, A.; VISHNY, R. W. Management Ownership and Market Valuation: an empirical analysis. J ournal of Financial Economics, Netherlands, p. 293-315, 1987.

PALEPU, K. More than a Fair Share: Principal-Principal Conflicts in Emerging Markets. Albuquerque: Southwestern Finance Association 2009.

PARKER, Martin et al. (Ed.). The Routledge companion to alternative organization. London: Routledge, 2014.

PATIN, R. P; MCNIEL, D. W. Benefit imbalances among credit union member groups: evidence of borrowerdominated, saver dominated and neutral behaviour?. Applied Economics, United Kingdom, v. 23, n. 4, p. 769$780,1991$.

PENG, M. W.; SAUERWALD, S. Corporate governance and principal-principal conflicts. Oxford: Oxford Handbook, 2012.

RENDERS, A.; GAEREMYNCK, A. Corporate Governance, Principal-Principal Agency Conflicts, and Firm Value in European Listed Companies. Corporate Governance: An International Review, United Kingdom, v. 20, n. 2, p. 125-143, 2012.

RUBIN, Geoffrey M. et al. A dynamic theory of the credit union. Annals of Operations Research, v. 205, n. 1, p. 29-53, 2013.

SAMPIERI, R. H.; COLLADO, C. F.; LUCIO, P. B. Metodologia de pesquisa. Tradução de Daisy Vaz de Moraes. New York: Mc Graw Hill, New York 2013.

SMITH, D. J.; A test for variant objective functions in credit unions. Applied Economics, United Kingdom, v. 18, n. 9, p. 959-970, 1986.

SOARES, M.; MELO SOBRINHO, A. Governança Cooperativa: Diretrizes e mecanismos para fortalecimento da governança em cooperativas de crédito. Brasília: Banco Central do Brasil, 2009.

SU, Y.; XU, D.; PHAN, P. H. Principal-Principal Conflict in the Governance of the Chinese Public Corporation. Management and Organization Review, United Kingdom, v. 4, n. 1, p. 17-38, 2008.

SPARROWE, Raymond.; MAYER, Kyle J. From the editors: Publishing in AMJ - Part 4: Grounding hypotheses. Academy of Management J ournal, United States, v. 54, n. 6, p. 1098-1102, 2011.

TAYLOR, R. A. The Credit Union as a Cooperative Institution. Review of Social Economy, United Kingdom, v. 29, n. 2, p. 207-217, 1971.

TAYLOR, R. A. Credit Unions and Economic Efficiency. Rivista Internationale di Scienze Economiche e Commerciali, v. 24, p. 239-247, 1977.

TAYLOR, R. A. Optimal Reserve Levels for Credit Unions. Rivista Internationale di Scienze Economiche e Commerciali, Padova, v. 26, p. 971-983, 1979.

TOSINI, M. F. C.; BASTOS, A. M. Governança Cooperativa: As funções de fiscalização e controle em cooperativas de crédito no Brasil. Revista de Contabilidade e Organizações USP, São Paulo, v. 2, n. 4, p. 75-89, dez. 2008. 
TRINDADE, L. Z:; BIALOSKORSKI NETO, S. Uma análise da separação entre a propriedade e a gestão nas cooperativas de crédito brasileiras. Revista de Contabilidade e Organizações, São Paulo, v. 6 n. 16, p.95-118, dez. 2012.

TRINDADE, L. Z.; BIALOSKORSKI NETO, S. Análise e Percep ção dos Custos das Práticas de Governança Corporativa: Um Estudo de Caso. Revista Contemporânea de Economia e Gestão, São Paulo, v. 12 n. 3, dez. 2014.

WALKER, M. C.; CHANDLER, G. G. On the Allocation of the Net Monetary Benefits of Credit Union Membership. Review of Social Economy, v. 35, n. 2, p. 159-168, 1977.

WARD, D.; FILATOTCHEV, I. Principal-Principal-Agency Relationships and the Role of External Governance. Managerial and Decision Economics, v. 31, p. 249-261, 2010.

WESTLEY, Glenn D., BRANCH, Brian (org.). Safe money: building effective credit unions in Latin America. Washington: Inter-American Development Bank and World Council of Credit Unions, 2000.

WHETTEN, David A. What constitutes a theoretical contribution? Academy of management review, v. 14, n. 4, p. 490-495, 1989.

WOAN, L. P. The Minority Shareholder's Statutory Exits. Singapore J ournal of Legal Studies, p. 184-196, 2007. WOOLDRIDGE, J. M. Introdução à econometria: Uma abordagem moderna. 4. ed. Thomson: 2011.

YOUNG, M. N. Corporate Governance in Emerging Economies: A Review of the Principal-Principal Perspective. Journal of Management Studies, v. 45, n. 1, jan. 2008.

ZYLBERSZTAJ N, D. Organização de cooperativas: desafios e tendências. Revista de Administração, São Paulo, v. 29, n. 3, p. 23-32, set. 1994.

ZYLBERSZTAJ N, D. Quatro estratégias fundamentais para cooperativas agrícolas. Working Paper no 02/017. FEA. São Paulo: USP, 2002. 